Lepr Rev (1992) 63, Supplement, 21s-22s

\title{
Summary of 'Estimation of the leprosy problem through health services data'
}

\author{
K JESUDASAN
}

When estimating the leprosy problem in countries and areas, often the only available data are gathered as part of routine health service data collection.

Reviews of country situations in the Western Pacific Region of the World Health Organization indicate that in spite of some drawbacks, routine health service data provide very useful information on the epidemiology of leprosy and can also be used to plan leprosy elimination programmes.

Estimation of the leprosy problem in countries is usually the basis for the following:

1 understanding the epidemiology of leprosy;

2 evaluation of the impact of leprosy control measures;

3 estimation of the problems of disability;

4 measuring the effectiveness of MDT on leprosy control, and

5 planning intervention for leprosy elimination.

\section{Epidemiological data collected}

Initially, the data for the calculation of the prevalence and incidence rates are collected. These rates by population units such as provinces or subdistricts are worked out to obtain a geographical profile of the leprosy situation.

Additional data on the age, sex, type, disability rates and contact factors provide information on the current status of the leprosy problem. It would be expected that in a high endemic area the prevalence and incidence would be high, the age group with leprosy would be younger with a predominance of PB cases and low deformity rate. However, much would depend on the effectiveness of case finding.

Analyses of secular trends over a period of time provide invaluable data on changes in the epidemiological situation of leprosy. Consistent changes validate observations made at different times.

\section{Operational information}

Then data on the operational aspects of leprosy control are collected. This includes information on the organizational structure of leprosy control, whether vertical, semivertical or integrated; case detection activities; health education; treatment and 
surveillance including relapses on monotherapy and MDT; bacteriological enumeration; hospitalization facilities; rehabilitation; training inputs; monitoring and supervision.

Analysis of operational information provides information on programme function. This information provides valuable clues to the estimation of the leprosy problem through data routinely available in most countries and provides programme managers with a rapid assessment. This has been demonstrated by experience gained in India, the Philippines, Papua New Guinea, Fiji, Western Samoa, Tonga, the Cook Islands and also Vanuatu. 